\title{
IDENTIFICATION OF MALASSEZIA SPECIES AND ITS ASSOCIATION WITH CLINICAL MANIFESTATIONS OF ATOPIC DERMATITIS
}

\author{
Tran Cam Van*, Nguyen Van Hieu**
}

\section{ABSTRACT}

Introduction: Atopic dermatitis is a chronic, recurrent inflammatory skin disease that is characterized by an eczematous reaction. Few studies have investigated fungi in the pathogenesis of atopic dermatitis, however, there are different about distribution of Malassezia species. Objectives: To indentificate of Malassezia species and its asociation with clinical manifestations in Vietnamese atopic dermatitis patient. Methods: 178 patients who were diagnosed with atopic dermatitis and had a postitive direct examination of Malassezia at the National hospital of dermatology and venereology between July 2019 and June 2020. Specimens were taken with cellotape, then stained in $20 \%$ of potassium hydroxit combined with Parker ${ }^{\mathrm{TM}}$ blue black ink. All patient who had postive test were cultured on SDA and mDixon. For fungal samples, we selected pure colonies with morphological characteristics of yeast as follows about $1 \mathrm{~cm}$ in diameter, round, cream or milky in color, smooth and glossy to detect the species. Results: From the samples of atopic dermatitis patients, we cultured and idenfified 41 cases. 5 species were found, in which M. globosa was the most common species, accounting for $39 \%$, followed by $M$. restricta (19.5\%), M. dermatis (17.1\%), M. furfur (17.1\%) and M. sympodialis (2.4\%). Conclusion: On the skin lesions of Vietnamese patients with atopic dermatitis, $M$. globosa was the most common species with $39.0 \%$.

\footnotetext{
* National Hospital of Dermatology and Venereology

** Hanoi Medical University

Responsible person: Tran Cam Van

Email: trancamvan.dl@gmail.com

Date of receipt: 30/6/2021

Date of scientific judgment: 31/72021

Reviewed date: 19/8/2021
}

\author{
Key words: Malassezia, Atopic dermatitis
}

\section{INTRODUCTION}

Atopic dermatitis (AD) is a chronic, recurrent inflammatory skin disease that is characterized by an eczematous reaction. In recent decades, atopic dermatitis prevalence has been increasing worldwide, estimated at $15-20 \%$ of children and 1-3\% of adults [1]. Many factors are involved in disease progression and severity, especially genetics, but the mechanism is still unclear. Dry skin and impaired skin barrier function play a major role responsing to triggers and external factors such as microorganisms. Using topical corticosteroids, immunomodulators, moisturizers and biological drugs can help treat disease, however, there are not effective. So, It is necessary to determine the role of some factors such as microorganisms, house dust or food.

Malassezia fungi is a yeast specialized to live on skin microflora. Few studies have investigated fungi in the pathogenesis of AD [1]. Nowicka et al (2019) detected 13 allergens from $M$. furfur and M. sympodialis [2]. Glatz et al research on 132 childrens and 67 adults, find a correlation between the fungi infection and the severity of disease [3]. However, there are different about distribution of Malassezis species. In Japan, Sugita et al (2003) showed that M. globosa and $M$. restricta are the common species with $93.8 \%$ and $87.5 \%$, respectively [4]. In 
Sweden, according to Falk et al (2005), the most species in lesion of AD patients was $M$. sympodialis with proportion with $46 \%$, following M. obtusa (30\%), M. globosa (28\%) [5]. Thus, seven species of Malassezia were detected in skin lesions of patients with $\mathrm{AD}$ [2].

This study aims to contribute to role of fungi infection in the $\mathrm{AD}$ by indentification of Malassezia species in Vietnamese AD patient.

\section{OBJECTIVES AND METHODS}

The study was performed in 178 patients who were diagnosed with $\mathrm{AD}$ and had a postitive direct examination of Malassezia at the National hospital of dermatology and venereology between July 2019 and June 2020.

Hanifin and Rijka's standard was used to diagnosis AD. Then, direct examination from scales on skin lesion was carried out. Specimens were taken with cellotape, then stained in $20 \%$ of potassium hydroxit combined with Parker $^{\mathrm{TM}}$ blue black ink.
After 30 mins, we examined by microscopy (40X and 100X magnification), evaluated the number of yeasts per a microscopic field as follows negative if from 0 to 4 cells, postitve $1+$ if from 5 to 14 cells, positive $2+$ if from $15-29$ cells, positive $3+$ if from 30 to 39 cells, positive $4+$ if above 40 cells. Direct examination microscopy results were considered positive when yeast cell and filament morphology was found or yeast cell morphology with a count of $3+$ or $4+$.

All patient who had postive test were cultured on SDA and mDixon, store in incubator at $32^{\circ} \mathrm{C}$ and $40^{\circ} \mathrm{C}$, followed up within one week. For fungal samples, we selected pure colonies with morphological characteristics of yeast as follows about $1 \mathrm{~cm}$ in diameter, round, cream or milky in color, smooth and glossy to detect the species.

\section{RESULTS}

From the samples of $\mathrm{AD}$ patients, we cultured and idenfified 41 cases, the following results:

Table 3. Culture results from scales of AD patient $(n=41)$

\begin{tabular}{|c|c|c|}
\hline Malassezia species & n & \% \\
\hline M. globosa & 16 & 39.0 \\
\hline M. restricta & 8 & 19.5 \\
\hline M. furfur & 7 & 17.1 \\
\hline M. dermatis & 7 & 17.1 \\
\hline M. sympodialis & 1 & 2.4 \\
\hline Malassezia spp. & 2 & 4.9 \\
\hline Total & 41 & 100 \\
\hline
\end{tabular}


No1\&2/2021 VIETNAM MEDICAL JOURNAL

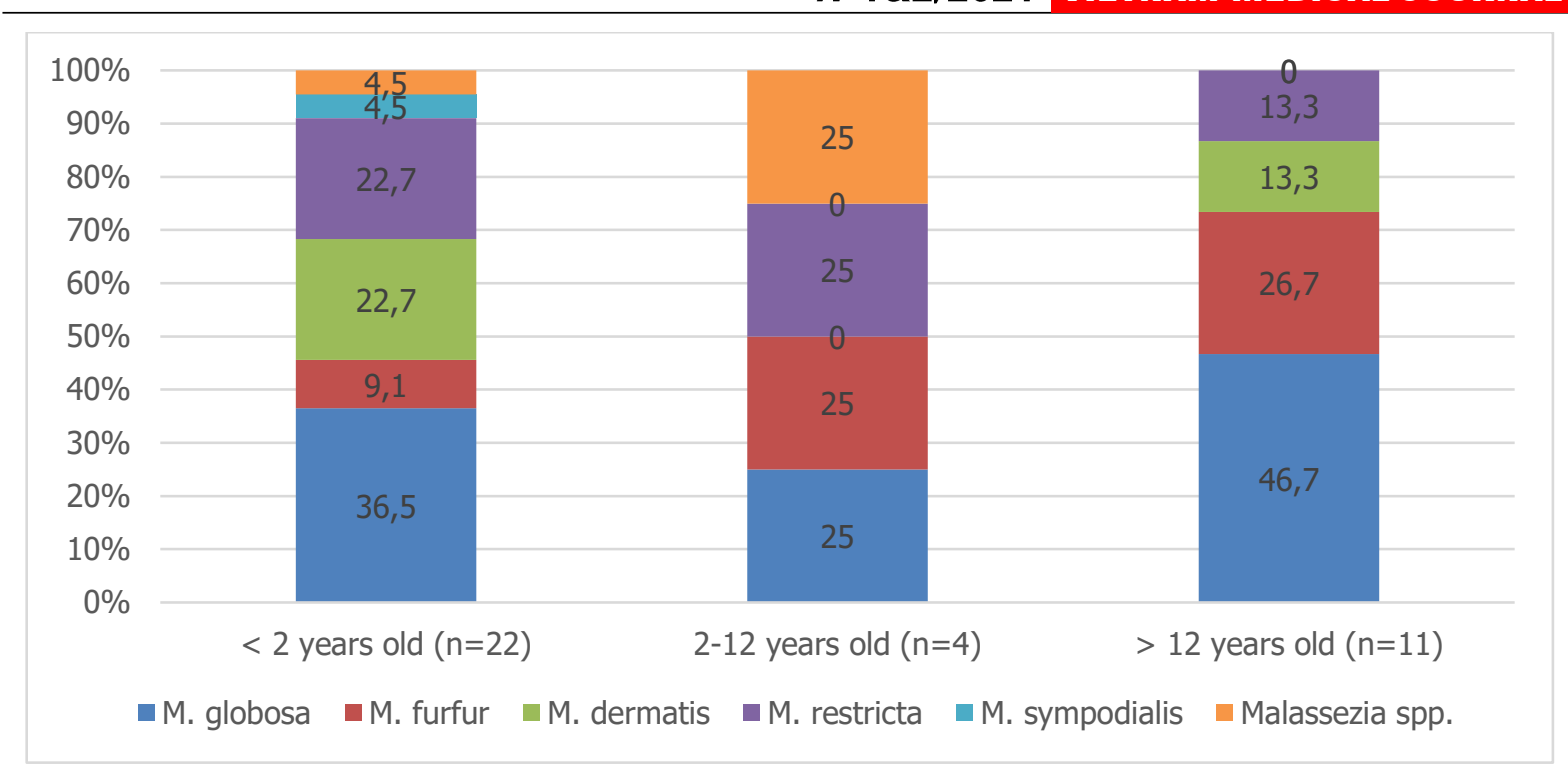

Figure 1. Distribution of Malassezia species by age group $(n=41)$

Table 4. Distribution of Malassezia species by sex $(\mathrm{n}=41)$

\begin{tabular}{|c|c|c|c|c|}
\hline \multirow{2}{*}{ Malassezia species } & \multicolumn{2}{|c|}{ Male } & \multicolumn{2}{c|}{ Female } \\
\cline { 2 - 5 } & $\mathbf{n}$ & $\mathbf{\%}$ & $\mathbf{n}$ & \% \\
\hline M. globosa & 12 & 41.4 & 4 & 33.3 \\
\hline M. restricta & 5 & 17.2 & 3 & 25.0 \\
\hline M. furfur & 4 & 13.8 & 3 & 25.0 \\
\hline M. dermatis & 6 & 20.7 & 1 & 8.3 \\
\hline M. sympodialis & 1 & 3.4 & 0 & 0 \\
\hline Malassezia spp. & 1 & 3.4 & 1 & 8.3 \\
\hline Total & 29 & 100 & 12 & 100 \\
\hline
\end{tabular}

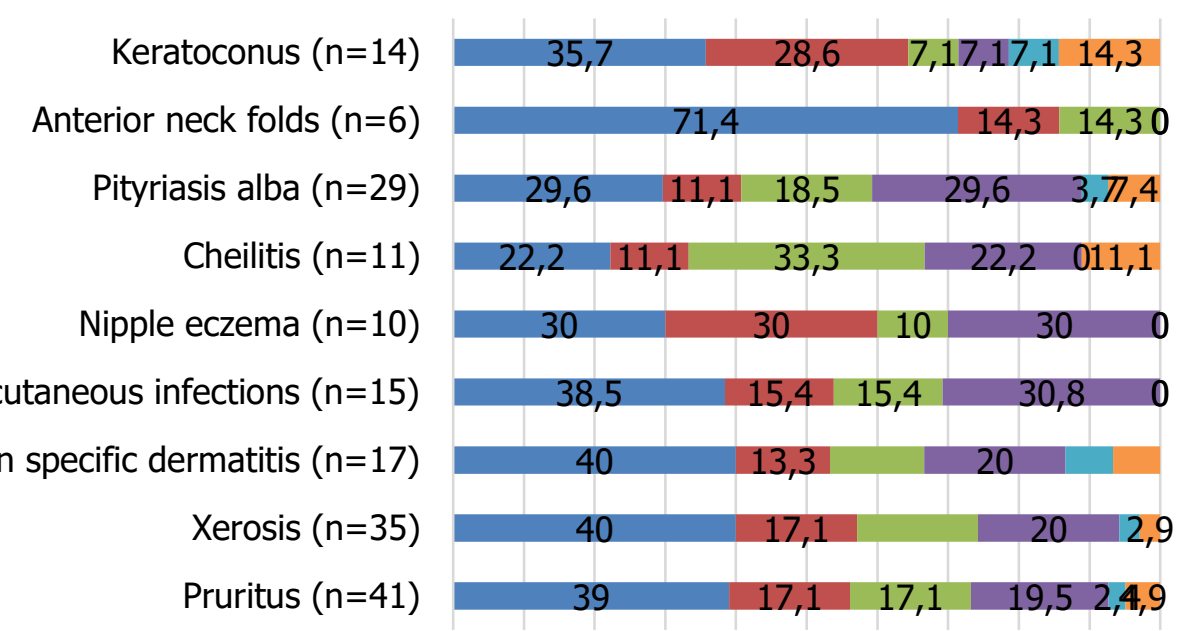

0\% $10 \% 20 \% 30 \% 40 \% 50 \% 60 \% 70 \% 80 \% 90 \% 100 \%$

$\square$ M. globosa $\square$ M. furfur $\square$ M. dermatis $\square$ M. restricta $\square$ M. sympodialis $\square$ Malassezia spp.

Figure 2. Distribution of Malassezia species according to clinical features $(n=41)$ 
Table 5. Distribution of Malassezia species according to disease progression and severity

\begin{tabular}{|c|c|c|c|c|c|c|c|}
\hline & $\begin{array}{c}\text { M. } \\
\text { globosa }\end{array}$ & $\begin{array}{c}\text { M. } \\
\text { restricta }\end{array}$ & $\begin{array}{c}\text { M. } \\
\text { furfur }\end{array}$ & $\begin{array}{c}\text { M. } \\
\text { dermatis }\end{array}$ & $\begin{array}{c}\text { M. } \\
\text { sympodialis }\end{array}$ & $\begin{array}{c}\text { Malassezia } \\
\text { spp. }\end{array}$ & Total \\
\hline Disease progression \\
\hline Acute & $6(60.0)$ & $2(20.0)$ & $\begin{array}{c}1 \\
(10.0)\end{array}$ & $1(10.0)$ & 0 & 0 & $10(100)$ \\
\hline Subacute & $7(30.4)$ & $4(17.4)$ & $\begin{array}{c}4 \\
(17.4)\end{array}$ & $6(26.1)$ & $1(4.3)$ & $1(4.3)$ & $23(100)$ \\
\hline Chronic & $3(37.5)$ & $2(25.0)$ & $\begin{array}{c}2 \\
(25.0)\end{array}$ & 0 & 0 & $1(12.5)$ & $8(100)$ \\
\hline Disease severity & $2(33.4)$ & $2(33.3)$ & $\begin{array}{c}2 \\
(33.3)\end{array}$ & 0 & 0 & 0 & $6(100)$ \\
\hline Mild & $14(40.0)$ & $6(17.1)$ & $\begin{array}{c}5 \\
(14.3)\end{array}$ & $7(20.0)$ & $1(2.9)$ & $2(5.7)$ & $34(100)$ \\
\hline Moderate
\end{tabular}

Table 6. Some factors related to species distribution of M. globosa $(\mathrm{n}=41)$

\begin{tabular}{|c|c|c|c|c|c|c|}
\hline \multirow{3}{*}{\multicolumn{2}{|c|}{ Characteristic }} & \multicolumn{4}{|c|}{ M. globosa } & \multirow{3}{*}{$\mathbf{p}$} \\
\hline & & \multicolumn{2}{|c|}{ Positive } & \multicolumn{2}{|c|}{ Negative } & \\
\hline & & $\mathbf{n}$ & $\%$ & $\mathbf{n}$ & $\%$ & \\
\hline \multirow[b]{2}{*}{ Age group } & $<2$ years old & 8 & 50.0 & 14 & 56.0 & \multirow{2}{*}{$>0.05$} \\
\hline & $\geq 2$ years old & 8 & 50.0 & 11 & 34.0 & \\
\hline \multirow{2}{*}{ Gender } & Male & 12 & 75.0 & 17 & 68.0 & \multirow{2}{*}{$>0.05$} \\
\hline & Female & 4 & 25.0 & 8 & 32.0 & \\
\hline \multirow{2}{*}{ Geography } & City & 4 & 25.0 & 8 & 32.0 & \multirow{2}{*}{$>0.05$} \\
\hline & Countryside & 12 & 75.0 & 17 & 68.0 & \\
\hline \multirow{2}{*}{$\begin{array}{c}\text { Disease } \\
\text { progession }\end{array}$} & Acute & 6 & 37.5 & 4 & 16.0 & \multirow{2}{*}{$>0.05$} \\
\hline & Subacute - Chronic & 10 & 62.5 & 21 & 84.0 & \\
\hline \multirow{2}{*}{$\begin{array}{l}\text { Disease } \\
\text { serverity }\end{array}$} & Mild & 2 & 12.5 & 4 & 16.0 & \multirow{2}{*}{$>0.05$} \\
\hline & Moderate & 14 & 87.5 & 21 & 84.0 & \\
\hline \multirow{2}{*}{$\begin{array}{c}\text { Direct } \\
\text { examination }\end{array}$} & yeast cell and filament & 3 & 18.8 & 2 & 8.0 & \multirow{2}{*}{$>0.05$} \\
\hline & yeast cell & 13 & 81.2 & 23 & 92.0 & \\
\hline & otal & 16 & 100 & 25 & 100 & -- \\
\hline
\end{tabular}

\section{DISCUSSION}

Our results show $M$. globosa was the most common species at $39.0 \%$, followed by $M$. restricta (19.5\%. In Japan, Sugita et al revealed both $M$. globosa and $M$. restricta were common species in $\mathrm{AD}$ with proportion with $93.8 \%$ and $87.5 \%$ [4]. In Sweden, according to Falk et al, the most species was M. sympodialis (46\%), following M. obtusa (30\%), M. globosa (28\%) [5]. Our results were similar to Sugita et al but different from Falk et al. We supposed that the distribution of species depends on climate as M. globosa ussually found in tropical climates. This is also consistent with the study of Nowicka et al [2]. The rate of fungal detection by culture with following species M. globosa (16 to $28 \%$ in Sweden), M. sympodialis (32 to $51 \%$ in Canada), M. restricta (3 to $22 \%$ in Korea), M. furfur (4 to $21 \%$ in Korea), M. slooffiae (3 
to $7 \%$ in Sweden), M. obtusa (10 to $30 \%$ in Canada and Sweden), M. dermatis (6,5\% in Korea). Two species only detect by PCR techniques are $M$. japonica $(58 \%)$ and $M$. yamatoensis (14\%). We detected 5 of these 7 species, except M. obtusa and M. slooffiae. Although there is a difference in prevalence among these study, $M$. globosa is a common species on the skin of AD patients in tropical countries, including Vietnam.

\section{* Age group}

In a study on healthy subject by Gupta et al, there was a diference in the distribution of Malassezia species between the age groups, especially, M. globosa was found in all age group, $M$. restricta was only found in group of 15 to 40 years old and M. sympodialis was not found in children [6]. Falk et al only detect three species included $M$. obtusa, $M$. globosa and M. slooffiae in adults [5]. Prohic demonstrate that $M$. globosa isolated in group of 36 to 50 years old, also $M$. restricta in group of 21 to 35 years old [7]. According to Nakashima et al recognized $M$. globosa and $M$. restricta were two common species assosiated with disease serverity [8]. Although, Glatz et al confirmed there was a correlation between Malassezia species and disease derverity in adults, also not children because lipids which need to growth of Malassezia in children is lower than adults [1].

\section{* Disease progression}

The detection rate of $M$. globosa in acute group was $60.0 \%$ higher than in chronic group and subacute group. Han et al study on 16 patient with chronic $\mathrm{AD}$ and determine four species included $M$. globosa, $M$. restricta, M. sympodialis and $M$. furfur [9]. In this study, M. globosa was the most frequently isolated species, but, $M$. furfur which was found allergens was the low rate.
Hans supposed $M$. globosa and $M$. restricta are asociated with the onset or exacerbation of $\mathrm{AD}$ because of their high frequency on the skin lesions. According to Johansson et al [10], acute skin lesions of $\mathrm{AD}$ is associated with Th2/Th0 cytokines, whereas, chronic skin lesions with Th1 cytokines. The author has demonstrated that $M$. furfur is involved in positive reactions, which often appear in acute skin lesions. In our study, there was one of seven patient with acute $\mathrm{AD}$ found $M$. furfur. Beside, each species of fungus has a suitable growth $\mathrm{pH}$ such as $M$. furfur at $\mathrm{pH}=8.0$, also $M$. globosa at $\mathrm{pH}=6.0$.

\section{* Disease serverity}

There was no difference in the distribution of Malassezia strains in the mild disease group. In the group of moderate disease, $M$. globosa was the highest proportion with $40 \%$. Cho et al found out that genotype (GT) 10 of M. globsa was related with the severity of AD [11]. Although the colonization of Malassezia depends on lipid composition, water content and skin surface $\mathrm{pH}, M$. globosa has a genotype that is closely related to the disease serverity. This is different from Rup et al [12]. The positive culture rate in group of SCORAD score under 40 points was higher than group of SCORAD score above 40 points. So, the author suggested this is related to the inflammatory process and chemical mediators with antifungal activity. We supposed that M. globosa may be associated with severe AD or exacerbation of AD.

\section{CONCLUSION}

On the skin lesions of Vietnamese patients with AD, M. globosa is the most common species with $39.0 \%$, followed by $M$. restricta $(19.5 \%)$. 
REFERENCES

1. Glatz M., Bosshard P., SchmidGrendelmeier P. (2017). The Role of Fungi in Atopic Dermatitis. Immunol Allergy Clin North Am, 37(1), 63-74.

2. Nowicka D., Nawrot U. (2019). Contribution of Malassezia spp. to the development of atopic dermatitis. Mycoses, 62(7), 588-596.

3. Glatz M., Buchner M., von Bartenwerffer W. et al (2015). Malassezia spp.-specific immunoglobulin $\mathrm{E}$ level is a marker for severity of atopic dermatitis in adults. Acta Derm Venereol, 95(2), 191-196.

4. Sugita T., Takashima M., Kodama M. et al (2003). Description of a new yeast species, Malassezia japonica, and its detection in patients with atopic dermatitis and healthy subjects. J Clin Microbiol, 41(10), 46954699.

5. Sandstrom Falk M.H., Tengvall Linder M., Johansson C. et al (2005). The prevalence of Malassezia yeasts in patients with atopic dermatitis, seborrhoeic dermatitis and healthy controls. Acta Derm Venereol, 85(1), 17-23.

6. Gupta A.K., Kohli Y. (2004). Prevalence of Malassezia species on various body sites in clinically healthy subjects representing different age groups. Med Mycol, 42(1), 3542.
7. Prohic A., Jovovic Sadikovic T., Krupalija-Fazlic M. et al (2016). Malassezia species in healthy skin and in dermatological conditions. International Journal of Dermatology, 55(5), 494-504.

8. Nakashima T., Niwano Y. (2012), Fungus as an Exacerbating Factor of Atopic Dermatitis, and Control of Fungi for the Remission of the Disease, INTECH Open Access Publisher.

9. Han S.H., Cheon H.I., Hur M.S. et al (2018). Analysis of the skin mycobiome in adult patients with atopic dermatitis. Exp Dermatol, 27(4), 366-373.

10. Johansson C., Eshaghi H., Linder M.T. et al (2002). Positive atopy patch test reaction to Malassezia furfur in atopic dermatitis correlates with a $\mathrm{T}$ helper 2-like peripheral blood mononuclear cells response. Journal of investigative dermatology, 118(6), 1044-1051.

11. Cho O., Saito M., Tsuboi R. et al (2013). Relationships among the genotypes of Malassezia globosa colonizing patients with atopic dermatitis, the clinical severity of the disease, and the level of specific $\operatorname{IgE}$ antibodies. J Clin Exp Dermatol Res, 4, 197.

12. Rup E., Skóra M., Krzyściak P. et al (2011). Distribution of Malassezia species in patients with atopic dermatitis - Quality assessment. Postepy Dermatologii I Alergologii, 28, 187-190. 